\title{
Local Orientation Smoothness Prior for Vascular Segmentation of Angiography
}

\author{
Wilbur C.K. Wong ${ }^{1}$, Albert C.S. Chung ${ }^{1}$, and Simon C.H. Yu ${ }^{2}$ \\ 1 Department of Computer Science, \\ The Hong Kong University of Science and Technology, \\ Clear Water Bay, Kowloon, HK \\ \{cswilbur, achung\}@cs. ust.hk \\ 2 Department of Diagnostic Radiology and Organ Imaging, \\ The Prince of Wales Hospital, Shatin, NT, HK \\ simonyu@cuhk.edu.hk
}

\begin{abstract}
We present a new generic method for vascular segmentation of angiography. Angiography is used for the medical diagnosis of arterial diseases. To facilitate an effective and efficient review of the vascular information in the angiograms, segmentation is a first stage for other post-processing routines. The method we propose uses a novel $a$ priori - local orientation smoothness prior - to enforce an adaptive regularization constraint for the vascular segmentation within the Bayes' framework. It aspires to segment a variety of angiographies and is aimed at improving the quality of segmentation in low blood flow regions. Our algorithm is tested on numerical phantoms and clinical datasets. The experimental results show that our method produces better segmentations than the maximum likelihood estimation and the estimation with a multi-level logistic Markov random field model. Furthermore, the novel algorithm produces aneurysm segmentations comparable to the manual segmentations obtained from an experienced consultant radiologist.
\end{abstract}

\section{Introduction}

Evolution in technology surrounding vascular imaging has brought radiologists to non-invasive imaging modalities that can provide accurate $3 \mathrm{D}$ vascular information quickly to a variety of patients. Vascular imaging helps the physician to define the character and extent of a vascular disease, thereby aiding diagnosis and prognosis. To facilitate an effective and efficient review of the vascular information in angiograms, segmentation is a first stage for other post-processing routines or analyses, such as visualization, volumetric measurement, quantitative comparison and image-guided surgery [1].

A variety of approaches have been proposed for vascular segmentation. For instance, authors in [2], 3] demonstrated that the expectation maximization (EM) algorithm and a maximum likelihood (ML) estimate can be used to segment vascular structures automatically with a proper statistical mixture model. The use of gradient information to drive evolving contours with the level set 
method and topologically adaptable surfaces to segment vasculature in the angiograms has been proposed in [4, [5], 6], 7]. Region-growing approaches to segmenting the angiograms with initial segmentations have been illustrated in [8], 9]. Alternatively, a tubular object has been used to model vessel segment in the angiograms explicitly for ridge transversal, multiscale analysis of vasculature and vessel diameter estimation [10, [11. A multiscale line enhancement filter has been applied to segment curvilinear structures in the angiograms 12 .

In a previous published work [3, we demonstrated a method to combine speed and phase information for vascular segmentation of phase-contrast (PC) magnetic resonance angiography 1 (MRA). Since phase images are only available in PC MRA, we introduce a novel generic framework for vascular segmentation of a variety of angiographies. The new method depends solely on a speed image to segment PC MRA and aspires to segment other angiographies such as timeof-flight (TOF) MRA and 3D rotational angiography (RA).

When blood flows along a vessel, because of the blood viscosity, frictional force slows down the flow near the vascular wall [13]. As such, the intensity values are low at the boundary of vessels in the angiograms. The inhomogeneous regions are a challenge if vascular segmentation is to be robust.

The method we propose uses a new smoothness prior to improve the quality of segmentation, particularly, at the low blood flow regions. The a priori, namely local orientation smoothness prior, exploits local orientation smoothness of the vascular structures to enforce an adaptive regularization constraint for robust vascular segmentation.

We expect the application of the a priori can be extended to different areas, e.g., image restoration with edge-preserving or coherent-enhancing capability (see [14], 15] and references therein), segmentation of non-medical images such as radar images [16] and object extraction from video [17].

In the next section, we present the Bayes' approach to segmenting the angiograms. We describe a robust method used to estimate local orientation from the images in Section 3. The implementation issues are outlined in Section 4 The experimental results on numerical phantoms and clinical datasets are given in Section 5, and conclusions are drawn in Section 6.

\section{Bayes' Approach to Segmenting Angiograms}

In this section, we formulate the vascular segmentation problem on the Bayes' framework. We discuss the estimation of the global likelihood probability and present the definition of the new a priori used to enforce an adaptive regularization constraint for the segmentation.

\subsection{Problem Formulation}

A vascular segmentation problem is regarded as a process to assign labels from a label set $\mathcal{L}=\{$ vessel, background $\}$ to each of the voxels indexed in

${ }^{1}$ Magnetic resonance angiography is one of most widely available vascular imaging techniques in a clinical environment. 
$\mathcal{S}=\{1, \ldots, m\}$, where $m$ is the total number of voxels in an angiogram $\boldsymbol{y}$. Let a vector $\boldsymbol{x}$ be a segmentation of the image $\boldsymbol{y}$, each element in the vector $\boldsymbol{x}$ can be regarded as a mapping from $\mathcal{S}$ to $\mathcal{L}$, i.e., $x_{i}: \mathcal{S} \rightarrow \mathcal{L}$. A feasible segmentation $\boldsymbol{x}$ is, therefore, in a Cartesian product $\Omega_{x}$ of the $m$ label sets $\mathcal{L}$. The set $\Omega_{x}$ is known as a configuration space. In the Bayes' framework, the optimal solution is given by a feasible segmentation $\boldsymbol{x}^{*}$ of the angiogram $\boldsymbol{y}$, which maximizes $a$ posteriori probability $p(\boldsymbol{x} \mid \boldsymbol{y}) \propto p(\boldsymbol{y} \mid \boldsymbol{x}) p(\boldsymbol{x})$ over the space $\Omega_{x}$ [18. The likelihood probability $p(\boldsymbol{y} \mid \boldsymbol{x})$ can be application-specific. It suggests the likelihood of a particular label assignment, based on the intensity values in the angiogram $\boldsymbol{y}$. Whereas, the prior probability $p(\boldsymbol{x})$ constrains the solution contextually.

In order to have a tractable constraint, the Markov random field (MRF) theory is used. By virtue of the Hammersley-Clifford theorem [19, the Gibbs distribution provides us with a practical way of specifying the joint probability - the prior probability $p(\boldsymbol{x})$ - of an MRF. The maximum a posteriori (MAP) estimate $\boldsymbol{x}^{*}$, therefore, becomes a minimum of the summation of the likelihood energy and prior energy functions over the configuration space $\Omega_{x}$,

$$
\boldsymbol{x}^{*}=\arg \min _{\boldsymbol{x} \in \Omega_{x}}(U(\boldsymbol{y} \mid \boldsymbol{x})+U(\boldsymbol{x})),
$$

where $U(\boldsymbol{y} \mid \boldsymbol{x})=-\log p(\boldsymbol{y} \mid \boldsymbol{x})$ is the likelihood energy function; and $U(\boldsymbol{x})=$ $\sum_{c \in \mathcal{C}} V_{c}(\boldsymbol{x})$ is the prior energy function, which is a sum of clique potentials $V_{c}(\boldsymbol{x})$ over all possible cliques in $\mathcal{C} \subseteq \mathcal{S}$ [18].

\subsection{Estimation of the Global Likelihood Probability}

In practice, because of the high complexity of the random variables $\boldsymbol{x}$ and $\boldsymbol{y}$, it is computationally intractable to calculate the likelihood energy $U(\boldsymbol{y} \mid \boldsymbol{x})$ from the negative $\log$-likelihood, $-\log p(\boldsymbol{y} \mid \boldsymbol{x})$. As such, we assume that the intensity value of each voxel $y_{i}$ is independent and identically distributed (i.i.d.). The calculation of the likelihood energy becomes tractable since the global likelihood probability can be determined by local likelihood probabilities and the likelihood energy function can be expressed as:

$$
U(\boldsymbol{y} \mid \boldsymbol{x})=-\sum_{i \in S} \log p\left(y_{i} \mid x_{i}\right) .
$$

\subsection{Local Orientation Smoothness Prior}

The new a priori is presented in this section. It exploits local orientation smoothness of the vascular structures and is used to enforce an adaptive regularization constraint for the vascular segmentation within the Bayes' framework. A smoothness constraint has been used to solve low level vision problems. Applications such as surface reconstruction, optical flow determination and shape extraction, demonstrate that this generic contextual constraint is a useful a priori to a variety of low level vision problems [18]. In the MRF framework, contextual 
constraint is expressed as the prior probability or the equivalent prior energy function $U(\boldsymbol{x})$ as given in Equation 1 .

Because of the blood viscosity, blood flows are low at the boundary of vessels [13. Using speed information alone cannot give satisfactory segmentation in low blood flow regions [3]. Therefore, in this paper, we propose a local orientation smoothness prior, aiming at improving the quality of segmentation at the low blood flow regions. The local orientation smoothness prior is expressed as follows:

$$
U(\boldsymbol{x})=\sum_{i \in \mathcal{S}} \sum_{j \in \mathcal{N}_{i}}\left(1-f\left(x_{i}\right)\right) f\left(x_{j}\right) g(i, j)\left(\beta_{1} h_{1}(i, j)+\beta_{2} h_{2}(i, j)\right),
$$

where $\mathcal{N}_{i}$ denotes a set of voxels neighboring the voxel $i$ with respect to a neighborhood system $\mathcal{N} \subseteq \mathcal{S} ; f$ is a mapping function defined as follows:

$$
f\left(x_{i}\right)=\left\{\begin{array}{l}
0, x_{i}=\text { background } \\
1, x_{i}=\text { vessel }
\end{array}\right.
$$

$g(i, j)$ measures the geometric closeness (Euclidean distance) between voxels $i$ and $j ; h_{1}(i, j)$ and $h_{2}(i, j)$ measure the linear (rank 1) and planar (rank 2) orientation similarities at voxel $i$ in respect of voxel $j$ respectively; $\beta_{1}$ and $\beta_{2}$ are positive weights, which need not sum to one and are used to control the influence of orientation smoothness in the interactions between the adjacent voxels. The idea of applying geometric closeness and similarity measures as constraints is similar to the one exploited in the bilateral filters [20]. In other words, the function $g$ in Equation 3 defines the structural locality, whereas the functions $h_{1}$ and $h_{2}$ quantify the structural orientation smoothness.

In this paper, the geometric closeness, $g$, and orientation similarity measures, $h_{1}$ and $h_{2}$, are Gaussian functions of the magnitude of the relative position vector of voxel $j$ from voxel $i,\left\|\boldsymbol{u}_{i j}\right\|$, and the orientation discrepancy, $\delta$, between voxels $i$ and $j$ respectively. The geometric closeness function is given as a decreasing function $g$ when the distance $\left\|\boldsymbol{u}_{i j}\right\|$ increases:

$$
g(i, j)=\exp \left(-\frac{\left\|\boldsymbol{u}_{i j}\right\|^{2}}{2 \sigma_{g}^{2}}\right)
$$

where $\boldsymbol{u}_{i j}$ is the relative position vector of voxel $j$ from voxel $i$ and the parameter $\sigma_{g}$ defines the desired geometric influence between neighboring voxels. The orientation similarity function $h_{k}$ is written as a decreasing function when the orientation discrepancy $\delta$ increases:

$$
h_{k}(i, j)=\exp \left(-\frac{\delta^{2}\left(\hat{\boldsymbol{u}}_{i j}, \hat{\boldsymbol{w}}_{k}\right)}{2 \sigma_{h}^{2}}\right),
$$

where $k=1$ and $k=2$ denote rank 1 and rank 2 orientation similarities respectively; the discrepancy function $\delta$ is defined as follows:

$$
\delta(\boldsymbol{u}, \boldsymbol{v})=1-\left|\boldsymbol{u}^{T} \boldsymbol{v}\right|
$$


$\hat{\boldsymbol{w}}_{1}$ depicts the principal direction of a linear orientation, while $\hat{\boldsymbol{w}}_{2}$ depicts one of the principal directions of a planar orientation'2; and the parameter $\sigma_{h}$ is chosen based on the desired amount of orientation discrepancy filtering amongst adjacent voxels.

In other words, the prior energy function in Equation 3 encourages piecewise continuous vessel label assignment in the segmentation. Vascular piecewise continuity is constrained by geometric closeness and orientation similarity measures. As long as voxels $i$ and $j$ are close enough, with similar rank 1 and/or rank 2 orientations, and the label assigned to voxel $j$ is vessel, i.e., $f\left(x_{j}\right)=1$, it is in favor of vessel label assignment to voxel $i$, i.e., $f\left(x_{i}\right)=1$. This is because we are minimizing the energy function in Equation 10. On the other hand, if the label assigned to voxel $j$ is background, i.e., $f\left(x_{j}\right)=0$, the prior energy vanishes and the label assignment to voxel $i$ is based solely on the likelihood energy.

\section{Estimating Local Orientation by Eigen Decomposition of Orientation Tensor}

Recall from Section 2 that we exploit the principal directions of linear and planar orientations, $\hat{\boldsymbol{w}}_{1}$ and $\hat{\boldsymbol{w}}_{2}$, to constrain the segmentation with the local orientation smoothness prior. In this section, we describe a robust method to estimate the principal directions. The estimation is obtained by an orientation tensor rather than a conventional Hessian matrix for the robustness to noise and performance reasons, see Section 5.1, for the performance comparisons of the two methods.

\subsection{Orientation Tensor}

The use of an orientation tensor for local structure description was first presented in Knutsson's work [21], which was motivated by the need to find a continuous representation of local orientation. Knutsson formulated the orientation tensor by combining outputs from a number of directional polar separable quadrature filters. A quadrature filter is constructed in the Fourier domain. It is a complex valued filter in the spatial domain, which can be viewed as a pair of filters: (1) symmetric (line filter) and (2) antisymmetric (edge filter). Further, it is orientation-specific and is sensitive to lines and edges that are orientated at the filter direction. In Knutsson's formulation, the orientation tensor $\mathbf{T}$ in a 3D space is defined as:

$$
\mathbf{T}=\sum_{k=1}^{6} q_{k}\left(\frac{5}{4} \hat{\boldsymbol{n}}_{k} \hat{\boldsymbol{n}}_{k}^{T}-\frac{1}{4} \mathbf{I}\right),
$$

where $q_{k}$ is the modulus of the complex valued response from a quadrature filter in the direction $\hat{\boldsymbol{n}}_{k}$ and $\mathbf{I}$ is the identity tensor. For further details see [21] or Chapter 6 in $[22]$.

\footnotetext{
${ }^{2}$ The orientation of a planar structure is depicted by two principal directions, which are orthogonal to its normal vector; as a planar structure can be seen as a series of linear structures, it is noted that, other than the vector $\hat{\boldsymbol{w}}_{2}, \hat{\boldsymbol{w}}_{1}$ depicts the other principal direction of the planar structure.
} 


\subsection{Estimating Local Orientation}

Estimation of local orientation is performed via eigen decomposition of the orientation tensor $\mathbf{T}$ at each voxel in an image 22. To calculate the tensor $\mathbf{T}$, the image should be convolved with the six quadrature filters. After the convolutions, there are six moduli of the complex valued filter responses associated with each voxel, $q_{k}, k=1,2, \ldots 6$. The tensor is computed as stated in Equation8.

Let $\lambda_{1}, \lambda_{2}$ and $\lambda_{3}$ be the eigenvalues of the tensor $\mathbf{T}$ in descending order $\left(\lambda_{1} \geq \lambda_{2} \geq \lambda_{3} \geq 0\right)$ and $\hat{\boldsymbol{e}}_{i}(i=1,2,3)$ are the corresponding eigenvectors respectively. The estimation of the local orientation can be one of the three cases as follows: (a) planar case: $\lambda_{1} \gg \lambda_{2} \simeq \lambda_{3}, \hat{\boldsymbol{e}}_{2}$ and $\hat{\boldsymbol{e}}_{3}$ are estimates to the principal directions of the planar structure; (b) linear case: $\lambda_{1} \simeq \lambda_{2} \gg \lambda_{3}$, $\hat{e}_{3}$ is an estimate to the principal direction of the linear structure; and (c) isotropic case: $\lambda_{1} \simeq \lambda_{2} \simeq \lambda_{3}$, no specific orientation. Therefore, we can approximate the principal directions of the linear and planar orientations $\left(\hat{\boldsymbol{w}}_{1}\right.$ and $\hat{\boldsymbol{w}}_{2}$ in Equation 6) with the eigenvectors $\hat{\boldsymbol{e}}_{3}$ and $\hat{\boldsymbol{e}}_{2}$ respectively.

\section{Implementation}

The proposed algorithm, summarized in Algorithm 11, consists of three parts. We discuss each of the three parts in Sections 4.1, 4.2 and 4.3 respectively.

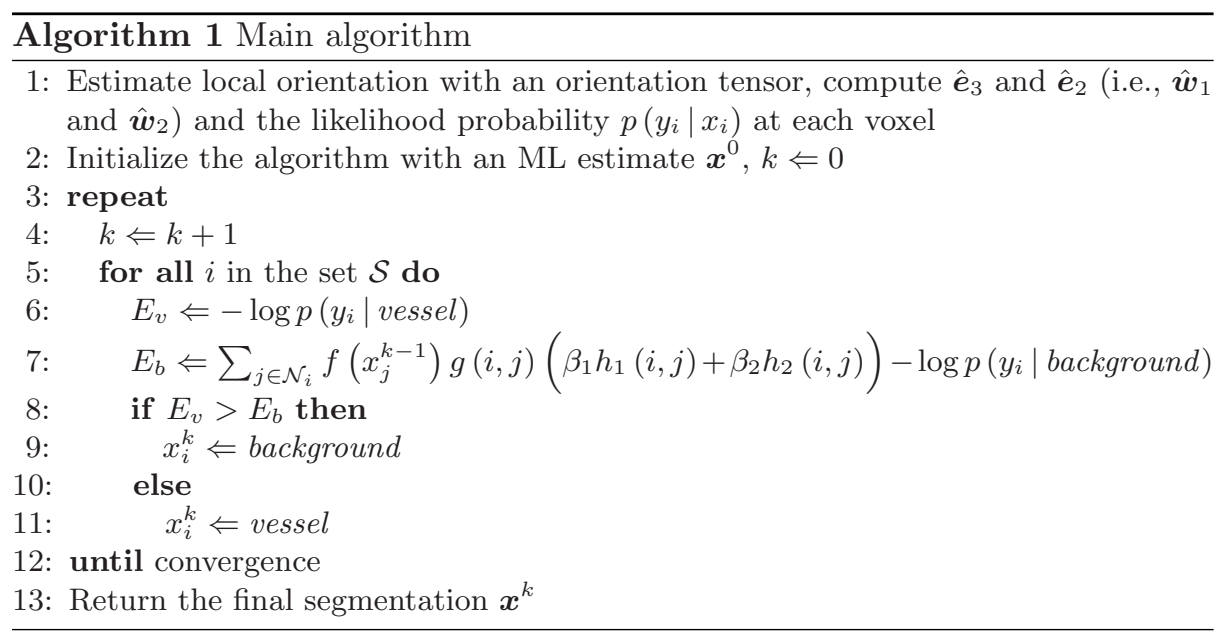

\subsection{Estimating Local Orientation}

To estimate the local orientation with an orientation tensor, as outlined in Section 3 six quadrature filters of a window size $5 \times 5 \times 5$, relative bandwidth $B$ equals 2 and center frequency $\rho$ equals $\frac{\pi}{2 \sqrt{2}}$ are used (relative bandwidth $B$ and 


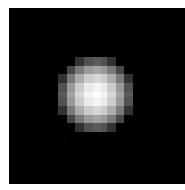

(a)

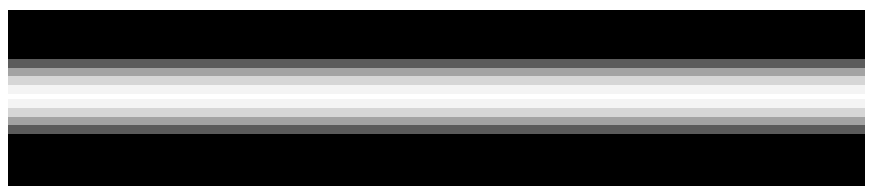

(b)

Fig. 1. Horizontal pipe phantom with a parabolic flow profile. (a) Cross section orthogonal to the pipe orientation, (b) central cross section along the pipe orientation

center frequency $\rho$ control the characteristics of the quadrature filters, see 22 . for further details). The 3D image is convolved with the filters to obtain six moduli of complex valued responses at each voxel. Then the orientation tensor is computed as given in Equation 8. The eigen decomposition of the orientation tensor is performed and the two vectors $\hat{\boldsymbol{e}}_{3}$ and $\hat{\boldsymbol{e}}_{2}$, which depict the principal directions of the linear and planar orientations, are obtained at each voxel.

\subsection{Likelihood Estimation and Algorithm Initialization}

As discussed in Section 2.1 the likelihood estimation can be application-specific. In this work, we use statistical mixture models to estimate the likelihood probabilities of the numerical phantoms and clinical datasets under the i.i.d. assumption (details are given in Sections 5.2 and 5.3 respectively).

To initialize the algorithm, an ML estimate is used. Given the likelihood probabilities are known, the initial segmentation $\boldsymbol{x}^{0}$ is obtained as follows:

$$
\boldsymbol{x}^{0}=\left\{\arg \max _{x_{i} \in \mathcal{L}} p\left(y_{i} \mid x_{i}\right) \mid \forall i \in \mathcal{S}\right\} .
$$

\subsection{Solution by Iterated Conditional Modes}

We use iterated conditional modes (ICM) 23] to solve the minimization problem in Equation 1 with deterministic local search for the following reasons: (1) the formulations of the likelihood energy and prior energy functions are entirely based on local information, see Sections 2.2 and 2.3 for details respectively; (2) our initial estimate of the truth segmentation can be very close to the optimal solution; and (3) the ICM optimization algorithm gives fast convergence to the solution and is simple to implement, which make it more attractive to timecritical medical applications than other optimization algorithms.

\section{Validation, Sensitivity Analyses, and Experiments}

In this section, the local orientation estimation (described in Section [3) is validated. Furthermore, sensitivity of the proposed algorithm is studied and experiments on numerical phantoms and clinical datasets are presented. 


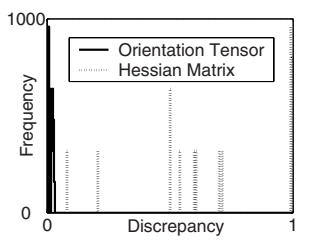

(a) Without noise

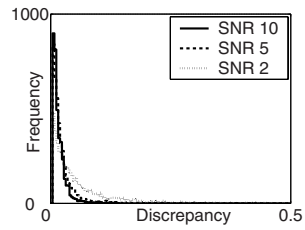

(b) With noise

Fig. 2. Orientation discrepancies between the estimated and the truth flow iso-surface normals. (a) Without noise; (b) with noise, the orientation tensor approach only

\subsection{Validation of Local Orientation Estimation}

As mentioned in Section 3] using an orientation tensor is not the only approach to estimating local orientation. A Hessian matrix (defined as in [12]) can also be used for the estimation (see [10], [11). In this section, we have conducted experiments to compare the performance of the two approaches.

A numerical horizontal pipe phantom with a parabolic flow profile 3 (peak flow magnitude equals 255) in a volume of size $100 \times 21 \times 21$ voxels has been built. The diameter of the pipe is 9 voxels, which is the average diameter of the major brain vessels in the clinical datasets. Figure 1 shows the noiseless pipe phantom.

We have compared the performance of the orientation tensor and Hessian matrix approaches (hereafter referred to as "OT" and "HESSIAN" respectively) on the noiseless phantom. Comparison is based on the orientation discrepancy (function $\delta$ in Equation 17) between the estimated and the truth flow iso-surface normal 4 . In OT, a $5 \times 5 \times 5$ filter window with relative bandwidth $B$ equals 2 and center frequency $\rho$ equals $\frac{\pi}{2 \sqrt{2}}$ has been used; a $3 \times 3$ Gaussian kernel with $\sigma=1$ has been employed for tensor averaging (for further details, see Chapter 6 in [22]). While a $5 \times 5 \times 5$ Gaussian smoothing kernel with $\sigma=\frac{5}{3}$ and a central finite difference approximation have been used in HESSIAN. Figure 2(a) shows the comparison between the two approaches. It is evident that OT gives a closeto-prefect orientation estimation. Conversely, owing to the use of second order derivatives and finite difference approximations, HESSIAN produces less than satisfactory results.

Furthermore, OT has been evaluated at different levels of additive white Gaussian noise. Signal-to-noise ratio (SNR) is defined as the ratio of the peak intensity value to the sample standard deviation of the noise. Figure 2(b) shows the discrepancy measures amongst SNR 2, 5 and 10 (on average, SNR is found to be about 5 in the clinical datasets). It is noted that OT is robust to noise. It gives, on average, a discrepancy value 0.05 , i.e., $18^{\circ}$ deviation from the truth flow iso-surface normals, even in the phantom corrupted by severe noise.

\footnotetext{
${ }^{3}$ A parabolic flow model is the simplest model to study blood flow in vessels 13 .

${ }^{4}$ We may think of the axis-symmetric flow in the pipe phantom as the sliding of a series of concentric tubes of fluid [13. The flow iso-surface normals are referred to as the surface normals of these tubes.
} 


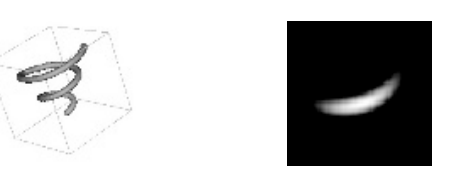

(a) Surface model

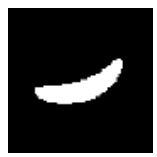

(e) Truth (b) Slice image

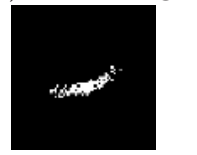

(f) ML estimation

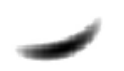

(c)

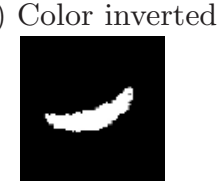

(g) Our algorithm

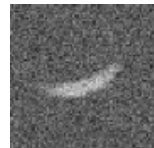

(d) SNR 5

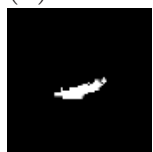

(h) MLL model

Fig. 3. Pipe phantom (radius equals 5 voxels) with the centerline aligned with a cubic B-spline curve in a 3D space. (a) 3D surface model, (b) a portion of a slice image, (c) color inverted slice image, (d) slice image with SNR equals 5, (e) truth segmentation, (f) ML estimation, (g) our estimation, (h) estimation with a MLL MRF model

\subsection{Sensitivity Analyses and Experiments on Numerical Phantoms}

There are four free parameters in the proposed algorithm, they are: $\sigma_{g}$ in Equation $5 \sigma_{h}$ in Equation 6. $\beta_{1}$ and $\beta_{2}$ in Equation 3 The parameters $\sigma_{g}$ and $\sigma_{h}$ define the desired geometric influence and amount of orientation discrepancy filtering amongst neighboring voxels respectively. Whereas, the two positive weights $\beta_{1}$ and $\beta_{2}$ control the influence of orientation smoothness in the interactions between the adjacent voxels.

Plausible values of the parameters $\sigma_{g}$ and $\sigma_{h}$ are suggested in this paragraph. To compromise between computational speed and robustness of the algorithm, a $3 \times 3 \times 3$ neighborhood system is used in the ICM algorithm. This leads to a justifiable choice to set $\sigma_{g}=1$. For the orientation discrepancy filtering, we suggest $\sigma_{h}=0.2$, this implies the algorithm has a $95 \%$ cut-off at discrepancy measure equals $2 \sigma_{h}=0.4$. In other words, the algorithm filters out neighboring voxels that are located outside the capture range of the filter, $\pm 53^{\circ}$ deviation from the estimated orientations, $\hat{\boldsymbol{w}}_{1}$ and $\hat{\boldsymbol{w}}_{2}$.

To understand the relationships between the two positive weights $\beta_{1}$ and $\beta_{2}$ and the sensitivity of the algorithm towards different object sizes and noise levels, a numerical phantom with a parabolic flow profile has been built. It is a pipe with the centerline aligned with a cubic B-spline curve in a $3 \mathrm{D}$ space. The phantom volume size is $128 \times 128 \times 128$ voxels. Figure 3 (a) shows the phantom as a $3 \mathrm{D}$ surface model.

The relationships between $\beta_{1}$ and $\beta_{2}$ with pipes, corrupted by additive white Gaussian noise, in different radii, 5, 2 and $1 \operatorname{voxel(s)~(in~the~clinical~datasets,~}$ vessel radius ranges from 1 voxel to 5 voxels) are shown in Figures 4 (a), 4(b) and 4 (c) respectively 5 . The SNR of the pipe phantoms is 5 . SNR is defined as in Section 5.1. The vertical axis of the graphs shows the Jaccard similarity coefficient (JSC) between the estimated and the truth segmentations. JSC is defined as the ratio of the intersection volume to the union volume of the two

\footnotetext{
${ }^{5}$ A Gaussian-uniform mixture model is used to estimate the likelihood probabilities.
} 


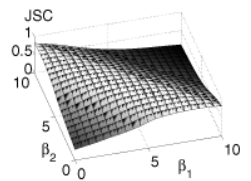

(a) 5 voxels radius

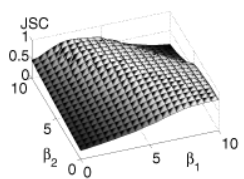

(b) 2 voxels radius

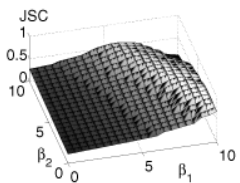

(c) 1 voxel radius

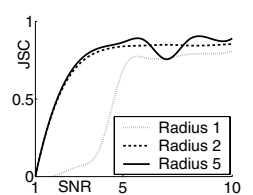

(d) Noise sensitivity

Fig. 4. Graphs from sensitivity analyses. (a)-(c) Relationship between $\beta_{1}$ and $\beta_{2}$, and (d) noise sensitivity of the algorithm with pipes in different radii

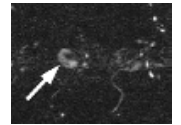

(a) Slice image

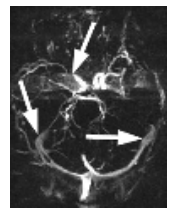

(e) MIP image

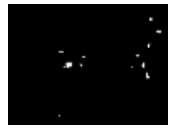

(b) ML estimation

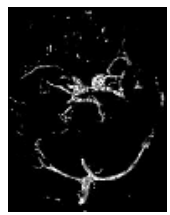

(f) ML estimation

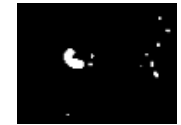

(c) Our algorithm

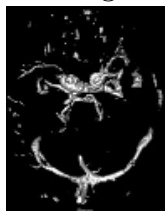

(g) Our algorithm

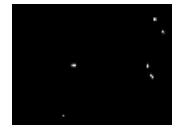

(d) MLL model

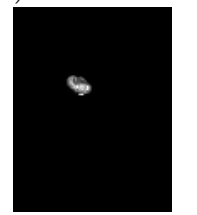

(h) Manual

Fig. 5. PC MRA dataset 1. (a) A slice image from the dataset; segmentation with (b) the ML estimation, (c) our algorithm, (d) the estimation with an MLL MRF model; (e) maximum intensity projection (MIP) image; volume rendered image of (f) segmentation with the ML estimation, (g) segmentation with our algorithm, (h) manual segmentation of the subregion that contains an aneurysm

given segmentations [24]. It is a similarity measure that maintains a balance between the sensitivity and specificity, and is used to quantify the accuracy of an estimated segmentation. JSC gives value 1 if the estimated segmentation equals the truth segmentation.

From the figures, it is noted that $\beta_{1}$ and $\beta_{2}$ complement each other. As the radius of the pipe decreases, $\beta_{1}$ contributes more to a better estimation; to recapitulate, $\beta_{1}$ controls the influence of the linear orientation smoothness in the interactions amongst neighboring voxels.

Figure 4(d) shows the noise sensitivity analysis of the algorithm with pipes in different radii. The weights $\beta_{1}$ and $\beta_{2}$ are set to the values that give maximum JSC as shown in Figures 4(a)-(c) for different pipe radii. It is shown that the algorithm is robust to noise over a wide range of object sizes. For small objects, i.e. 1 voxel in radius, the algorithm can give a satisfactory estimation if SNR $\geq 5$ (The average SNR in the clinical datasets is found to be 5 ).

Figures [3(f), 3)(g) and 3(h) show the segmentations obtained with the ML estimation, our algorithm and the estimation with a multi-level logistic (MLL) MRF model respectively. It is indicated that our algorithm produces satisfactory segmentation of the pipe (JSC $=0.88$ ) contrary to the ML estimation (JSC $=0.29)$ and the estimation with the MLL MRF model $(\mathrm{JSC}=0.26)$. 


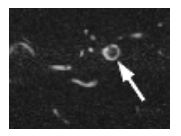

(a) Slice image

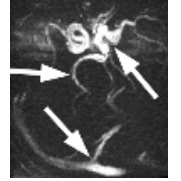

(e) MIP image

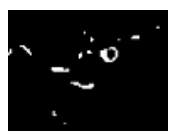

(b) ML estimation

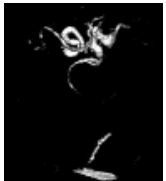

(f) ML estimation

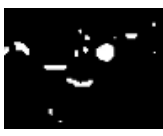

(c) Our algorithm

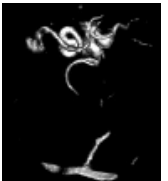

(g) Our algorithm

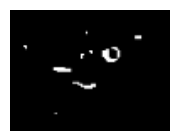

(d) MLL model

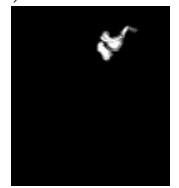

(h) Manual

Fig. 6. PC MRA dataset 2. Figure arrangement is identical to Figure[5]

\subsection{Experiments on Clinical Datasets}

The proposed segmentation algorithm has been applied to two clinical datasets. They were obtained from the Department of Diagnostic Radiology and Organ Imaging at the Prince of Wales Hospital, Hong Kong. The two PC MRA intracranial scans were acquired from a Siemens $1.5 \mathrm{~T}$ Sonata imager. The data volume is $256 \times 176 \times 30$ voxels with a voxel size of $0.9 \times 0.9 \times 1.5 \mathrm{~mm}^{3}$.

The experimental results are shown in Figures 5 and [6] It is evident that parts of the vascular structures in the low blood flow regions, i.e., the boundary of vessels and the aneurysm: 6 (indicated by the arrows), are left out in the segmentations produced by the ML estimation and the estimation with the MLL MRF model. On the contrary, our algorithm gives satisfactory segmentations in those regions.

Furthermore, our method produces aneurysm segmentations comparable to the manual segmentations obtained from a local consultant radiologist who has $>15$ years' clinical experience in endovascular neurosurgery (on average, JSC $=0.73$ for our algorithm versus JSC $=0.41$ for the ML estimation 7 ). See Figures 5(h) and 6(h) for the volume rendered image 8 of the manual segmentations for comparisons. It is observed that there is a large improvement in segmentation using our algorithm, especially for the vessels with low intensity values.

One may find that a few small vessels are left out in the segmentations produced by our algorithm showed in Figures 5(g) and 6(g). According to the radiologists' feedback, the segmentations obtained with our algorithm are good enough for clinical applications. Small vessels with diameter $<3$ voxels are not their current primary interest in this work.

In the experiments, the same set of parameter values is used for the segmentations of different clinical datasets. The parameter values are: $\sigma_{g}=1, \sigma_{h}=0.2$ and $\beta_{1}=\beta_{2}=3.5$. The Maxwell-Gaussian-uniform (MGU) mixture model is

\footnotetext{
${ }^{6}$ An aneurysm is an abnormal local dilatation of blood vessel.

7 The JSC values are computed within the subregions that contain the aneurysms with the manual segmentations treated as the truth segmentations.

${ }^{8}$ Volume rendering is performed using the Visualization Toolkit (www.vtk.org).
} 
employed to estimate the likelihood probabilities as suggested in [3]. On average, the algorithm takes $42 \mathrm{~s}$, needs $<20$ iterations to converge and consumes $<100 \mathrm{MB}$ of memory to segment the two PC MRA datasets on a $2.66 \mathrm{GHz} \mathrm{PC}$.

\section{Conclusions}

We have presented a new generic method for vascular segmentation of angiography. Our method uses a novel smoothness prior that exploits local orientation smoothness of the vascular structures to improve the quality of segmentation at low blood flow regions. The a priori is expressed as a function of geometric closeness and orientation similarity measures. Furthermore, we have described a method to estimate the local orientation with an orientation tensor. The experimental results have indicated that the method is more robust than that with a conventional Hessian matrix, and is capable of a more accurate orientation estimation.

Our algorithm has been applied to the numerical phantoms and clinical datasets. The experimental results have shown that the new method produces segmentations better than the maximum likelihood estimation and the estimation with the multi-level logistic Markov random field model. Moreover, the aneurysm segmentations obtained with our method are comparable to the manual segmentations produced by an experienced consultant radiologist.

In this work, we have demonstrated an application of the local orientation smoothness prior to medical image segmentation. We expect the use of the $a$ priori to be applicable to different areas such as image restoration with edgepreserving or coherent-enhancing capability, non-medical image segmentation and object extraction from video.

Acknowledgments. This work is supported in part by the Hong Kong Research Grants Council (HK RGC) under grants HKUST6209/02E and DAG01/02.EG04, and the Sino Software Research Institute (SSRI) under grant SSRI01/02.EG22. The authors wish to thank Prof. C.-F. Westin for the fruitful discussions, and providing MATLAB codes on the construction of the quadrature filters at the early stage of the software development. They would also like to thank Craig C. W. Jo and the anonymous reviewers for their perceptive comments, which have significantly improved the paper.

\section{References}

1. Suri, J.S., Liu, K., Reden, L., Laxminarayan, S.: A review on MR vascular image processing algorithms: Acquisition and prefiltering: Part I. IEEE Trans. Inform. Technol. Biomed. 6 (2002) 324-337

2. Wilson, D.L., Noble, J.A.: An adaptive segmentation algorithm for time-of-flight MRA data. IEEE Trans. Med. Imag. 18 (1999) 938-945

3. Chung, A.C.S., Noble, J.A., Summers, P.: Fusing speed and phase information for vascular segmentation of phase contrast MR angiograms. MedIA 6 (2002) 109-128 
4. Lorigo, L.M., Faugeras, O., Grimson, W.E.L., Keriven, R., Kikinis, R., Westin, C.F.: Co-dimension 2 geodesic active contours for MRA segmentation. In: IPMI. (1999) 126-139

5. Wang, K.C., Dutton, R.W., Taylor, C.A.: Improving geometric model construction for blood flow modeling. IEEE Eng. Med. Biol. Mag. 18 (1999) 33-39

6. Westin, C.F., Lorigo, L.M., Faugeras, O., Grimson, W.E.L., Dawson, S., Norbash, A., Kinkinis, R.: Segmentation by adaptive geodesic active contours. In: MICCAI. (2000) 266-275

7. McInerney, T., Terzopoulos, D.: Medical image segmentation using topologically adaptable snakes. In: CVRMed. Volume 905 of LNCS., Springer-Verlag Berlin (1995) 92-101

8. Masutani, Y., Schiemann, T., Hohne, K.H.: Vascular shape segmentation and structure extraction using a shape-based region-growing model. In: MICCAI. (1998) 1242-1249

9. Parker, D.L., Chapman, E., Roberts, J.A., Alexander, A.L., Tsuruda, J.S.: Enhanced image detail using continuity in the MIP Z-buffer: Applications to magnetic resonance angiography. JMRI 11 (2000) 378-388

10. Aylward, S., Bullitt, E.: Initialization, noise, singularities, and scale in height ridge traversal for tubular object centerline extraction. IEEE Trans. Med. Imag. 21 (2002) 61-75

11. Krissian, K., Maladain, G., Vaillant, R., Trousset, Y., Ayache, N.: Model-based multiscale detection of 3D vessels. In: CVPR. (1998) 722-727

12. Sato, Y., Nakajima, S., Shiraga, N., Atsumi, H., Yoshida, S., Koller, T., Gerig, G., Kikinis, R.: 3D multi-scale line filter for segmentation and visialization of curvilinear structures in medical images. MedIA 2 (1998) 143-168

13. Fung, Y.C.: Biomechanics: Circulation. Second edn. Springer-Verlag (1996)

14. Barash, D.: A fundamental relationship between bilateral filtering, adaptive smoothing, and the nonlinear diffusion equation. PAMI 24 (2002) 844-847

15. Weickert, J.: Coherence-enhancing diffusion filtering. IJCV 31 (1999) 111-127

16. Mignotte, M., Collet, C., Perez, P., Bouthemy, P.: Sonar image segmentation using an unsupervised hierarchical MRF model. IEEE Trans. Image Process. 9 (2000) $1216-1231$

17. Cui, Y.T., Huang, Q.: Character extraction of license plates from video. In: CVPR. (1997) 502-507

18. Li, S.Z.: Markov random field modeling in image analysis. Springer-Verlag Tokyo (2001)

19. Besag, J.: Spatial interaction and the statistical analysis of lattice systems (with discussion). JRSS, Series B (Methodological) 36 (1974) 192-236

20. Tomasi, C., Manduchi, R.: Bilateral filtering for gray and color images. In: ICCV. (1998) 839-846

21. Knutsson, H.: Representing local structure using tensors. In: The 6th Scandinavian Conf. on Image Analysis. (1989) 244-251

22. Granlund, G., Knutssan, H.: Signal processing for computer vision. Kluwer Academic Publishers (1995)

23. Besag, J.: On the statistical analysis of dirty pictures. JRSS, Series B (Methodological) 48 (1986) 259-302

24. Leemput, K.V., Maes, F., Vandermeulen, D., Colchester, A., Suetens, P.: Automated segmentation of MS lesions from multi-channel MR images. In: MICCAI. (1999) 11-21 